\title{
Antibacterial Effect of Thiosulfinates on Multiresistant Strains of Bacteria Isolated from Patients with Cystic Fibrosis
}

\author{
V. V. Kulikova', M. Yu. Chernukha², E. A. Morozova', S. V. Revtovich', A. N. Rodionov ${ }^{1}$, \\ V. S. Koval' ${ }^{1}$ L. R. Avetisyan², D. G. Kuliastova², I. A. Shaginyan², T. V. Demidkina1* \\ ${ }^{1}$ Engelhardt Institute of Molecular Biology of the Russian Academy of Sciences, Vavilova Str., 32, \\ Moscow, 119991, Russia \\ ${ }^{2}$ N.F.Gamaleya National Research Centre of Epidemiology and Microbiology, Ministry of Health of \\ Russian Federation, Gamaleya, Str., 18, Moscow, 123098, Russia \\ "E-mail: tvd@eimb.ru, tvdemidkina@yandex.ru \\ Received October 11, 2017; in final form June 26, 2018 \\ Copyright @ 2018 Park-media, Ltd. This is an open access article distributed under the Creative Commons Attribution License, which permits \\ unrestricted use, distribution, and reproduction in any medium, provided the original work is properly cited.
}

\begin{abstract}
The multiresistance of $A$. ruhlandii 155B, B. cenocepacia 122, and P. aeruginosa 48B strains isolated from patients with cystic fibrosis was established. The antibacterial effect of allicin, dimethyl thiosulfinate, and dipropyl thiosulfinate on multidrug-resistant strains was shown. Thiosulfinates can have both bacteriostatic and bactericidal effects depending on the microorganism and the concentration. The studied thiosulfinates may be candidates for the development of alternative antibiotic drugs to treat infections caused by multidrug-resistant pathogens.
\end{abstract}

KEYWORDS allicin, antibacterial activity, cystic fibrosis, methionine $\gamma$-lyase, thiosulfinates.

ABBREVIATIONS MGL - methionine $\gamma$-lyase, MIC - minimum inhibitory concentration, MBC - minimum bactericidal concentration.

\section{INTRODUCTION}

The emergence of novel approaches to the development of effective antibacterial drugs is of utmost relevance because of the wide spread of antibiotic-resistant strains of bacteria. Multidrug-resistant microorganisms cause nosocomial infections, which can be the origin of complications in weakened patients. The chronic pulmonary infection caused by the association of such pathogens as Pseudomonas aeruginosa, Staphylococcus aureus, Burkholderia cepacia complex, etc. in patients with cystic fibrosis [1] is a serious problem associated with the formation of multi-resistant strains of microorganisms as a result of prolonged antibiotic therapy, which renders further antibiotic therapy ineffective.

Thiosulfinates are found in plants of the genus $\mathrm{Al}$ lium and have an antimicrobial effect [2]. The antibacterial effect of allicin, the main thiosulfinate contained in garlic, is due to the combination of a reduced cellular glutathione level and inactivation of key metabolic enzymes as a result of the modification of their thiol groups [3, 4]. Since allicin, which oxidizes the thiol groups of enzymes and proteins, has many targets within the cell, it, alongside with other thiosulfinates, is unlikely to cause resistance [5].

Alliinase [EC 4.4.1.4] of the plants of the genus $A l$ lium catalyzes the decomposition of sulfoxides of the
S-substituted analogues of $L$-cysteine to give rise to thiosulfinates. We have shown that thiosulfinates can be obtained using methionine $\gamma$-lyase (MGL, [EC 4.4.1.11]) (Scheme). Thiosulfinates formed by the cleavage of S-allyl-L-cysteine, S-methyl-L-cysteine, and S-ethyl-L-cysteine sulfoxides catalyzed by both wild-type MGL and its more efficient mutant form, $\mathrm{C} 115 \mathrm{H}$, inhibit the growth of Gram-positive and Gram-negative bacteria [6], including $P$. aeruginosa isolated from murine intestine [7].

The aim of the current work was to study the antibacterial effect of thiosulfinates obtained by $\beta$-elimination of three S-substituted L-cysteine sulfoxides (Scheme) catalyzed by $\mathrm{C} 115 \mathrm{H}$ MGL on multidrug-resistant strains of the Gram-negative bacteria Achromobacter ruhlandii 155B, B. cenocepacia 122, and P. aeruginosa 48B isolated from patients with cystic fibrosis.

\section{EXPERIMENTAL}

Isolation of the enzyme, determination of its activity, synthesis of S-substituted L-cysteine sulfoxides, and production of thiosulfinates were carried out as previously described [6]. The concentrations of thiosulfinates were determined according to [8].

The antibacterial activity of thiosulfinates was determined by the two-fold serial dilution and agar diffusion method. 

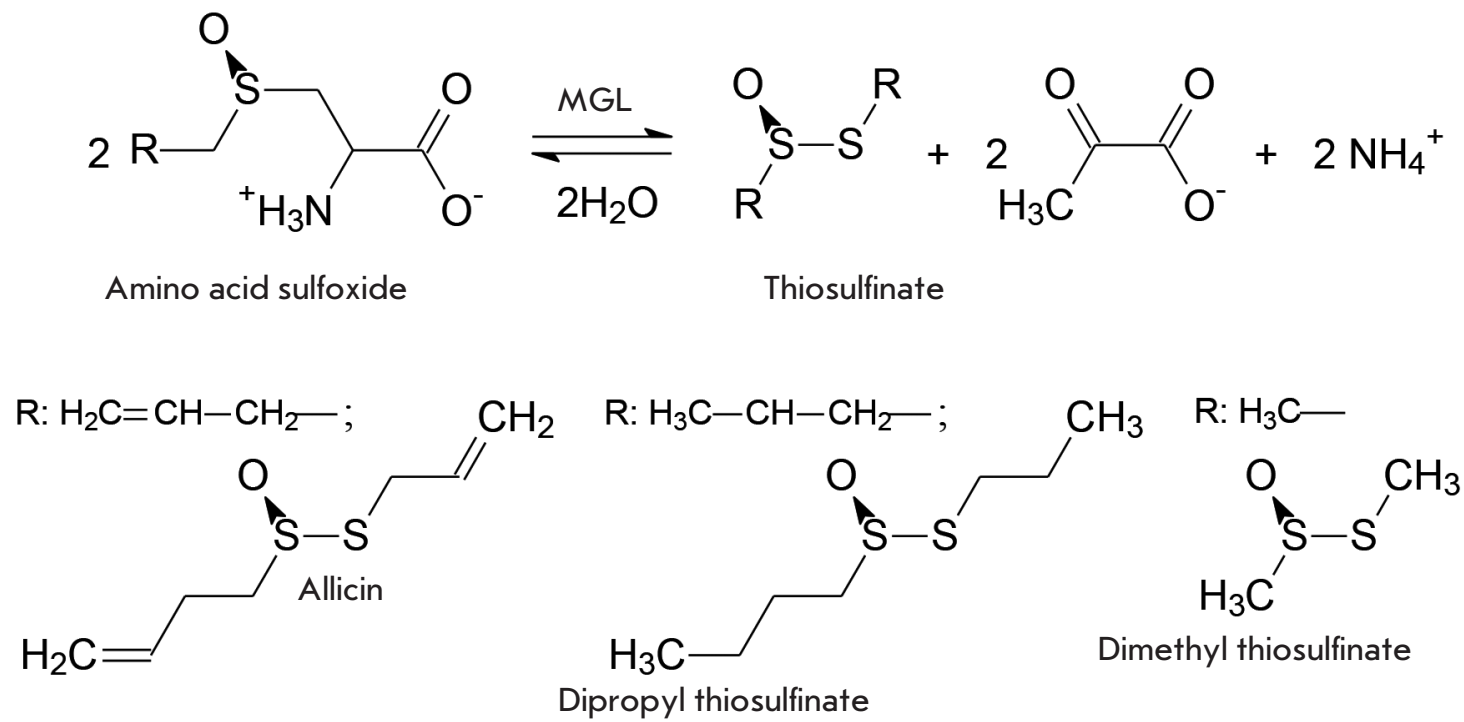

Dipropyl thiosulfinate

Scheme 1. $\beta$-elimination reaction of S-substituted $L$-cysteine sulfoxides

When determining the antibacterial activity of thiosulfinates by the method of two-fold serial dilutions [9], we used the Mueller-Hinton broth. Strains were cultivated at $10^{5} \mathrm{CFU} / \mathrm{ml}$ and supplemented with agents at concentrations ranging from 1 to $0.0039 \mathrm{mg} / \mathrm{ml}$, followed by inoculation into a dense growth medium (medium no. 1 for P. aeruginosa 48B and blood agar for A. ruhlandii $155 \mathrm{~B}$ and B. cenocepacia 122 ).

The antibacterial activity of the agents on the solid growth medium was determined at a concentration varied from 2 to $0.05 \mathrm{mg} / \mathrm{ml}$ by inoculating diluted strains $\left(10^{4}\right.$ to $\left.10^{7} \mathrm{CFU} / \mathrm{ml}\right)$ into Mueller-Hinton agar using the disc diffusion method and by directly applying the test samples at a volume of $10 \mu \mathrm{l}$.

Strain resistance to standard antibiotics prescribed to treat cystic fibrosis was determined by the serial dilution method according to the clinical recommendations on the threshold MIC values for each antibiotic [10].

The antibacterial efficacy of the thiosulphinates and antibiotics was compared using the disc diffusion method; strains were inoculated from the diluted cultures $\left(10^{6} \mathrm{CFU} / \mathrm{ml}\right)$ into Mueller-Hinton agar.

The duration of incubation on the solid growth medium was 24-48 $\mathrm{h}$ for all the experiments.

Strains A. ruhlandii 155B, B. cenocepacia 122, and $P$. aeruginosa 48B isolated from patients with cystic fibrosis and stored in the culture collection of the Laboratory of Molecular Epidemiology of Nosocomial Infections at the N.F.Gamaleya National Research Centre of Epidemiology and Microbiology (Ministry of Health of the Russian Federation) were used in this study.

\section{RESULTS AND DISCUSSION}

The antibacterial activities of allicin, dimethyl, and dipropyl thiosulfinate against the A. ruhlandii 155B, B. cenocepacia 122 , and $P$. aeruginosa $48 \mathrm{~B}$ strains isolated from patients with cystic fibrosis were determined (Table 1). The differences in the nature and degree of the antimicrobial effect of the thiosulfinates were revealed.

Allicin and dimethyl thiosulfinate turned out to exhibit the strongest effect against $B$. cenocepacia 122 and $P$. aeruginosa 48B, while dipropyl thiosulfinate was less active.

Table 1. The MIC and MBC values for thiosulfinates

\begin{tabular}{|c|c|c|c|}
\hline \multirow{2}{*}{$\begin{array}{l}\text { Bacterial } \\
\text { strain }\end{array}$} & \multirow{2}{*}{ Thiosulfinate } & MIC & MBC \\
\hline & & \multicolumn{2}{|c|}{$\mathrm{mg} / \mathrm{ml}$} \\
\hline \multirow{3}{*}{$\begin{array}{l}\text { A. ruhlandii } \\
\text { 155B }\end{array}$} & Allicin & 0.50 & 1 \\
\hline & Dimethyl thiosulfinate & $2.00 *$ & - \\
\hline & Dipropyl thiosulfinate & $2.00 *$ & - \\
\hline \multirow{3}{*}{$\begin{array}{c}\text { B. cenocepacia } \\
122\end{array}$} & Allicin & 0.03 & $\geq 0.03^{* *}$ \\
\hline & Dimethyl thiosulfinate & 0.03 & $\geq 0.03^{* *}$ \\
\hline & Dipropyl thiosulfinate & 0.25 & 0.5 \\
\hline \multirow{3}{*}{$\begin{array}{c}\text { P. aeruginosa } \\
48 \mathrm{~B}\end{array}$} & Allicin & 0.06 & 1 \\
\hline & Dimethyl thiosulfinate & 0.06 & - \\
\hline & Dipropyl thiosulfinate & 0.50 & - \\
\hline
\end{tabular}

Note. "-" - no bactericidal effect.

*The data were obtained in the experiment on determining antibacterial activity on a solid growth medium using the disc diffusion method.

**But not exceeding 0.06 . 
Table 2. Antibacterial efficacy of thiosulfinates at different cell concentrations

\begin{tabular}{|c|c|c|c|c|c|c|c|c|c|}
\hline \multirow{4}{*}{ Bacterial strain } & \multirow{4}{*}{ Thiosulfinate } & \multicolumn{8}{|c|}{ Diameter of inhibition zones $(\mathrm{mm})$ at cell concentration, $\mathrm{CFU} / \mathrm{ml}$} \\
\hline & & $10^{4}$ & $10^{5}$ & $10^{6}$ & $10^{7}$ & $10^{4}$ & $10^{5}$ & $10^{6}$ & $10^{7}$ \\
\hline & & \multicolumn{8}{|c|}{ and thiosulfinate concentration* $\mathrm{mg} / \mathrm{ml}$} \\
\hline & & \multicolumn{4}{|c|}{2} & \multicolumn{4}{|c|}{0.4} \\
\hline \multirow{3}{*}{ A. ruhlandii $155 \mathrm{~B}$} & Allicin & 30 & 30 & 30 & 30 & 0 & 0 & 0 & 0 \\
\hline & Dimethyl thiosulfinate & 30 & 30 & 30 & 30 & 0 & 0 & 0 & 0 \\
\hline & Dipropyl thiosulfinate & 30 & 30 & 30 & 30 & 0 & 0 & 0 & 0 \\
\hline \multirow{3}{*}{ B. cenocepacia 122} & Allicin & 25 & 25 & 25 & 25 & 0 & 0 & 0 & 0 \\
\hline & Dimethyl thiosulfinate & 25 & 25 & 25 & 25 & 0 & 0 & 0 & 0 \\
\hline & Dipropyl thiosulfinate & 20 & 20 & 20 & 20 & 0 & 0 & 0 & 0 \\
\hline \multirow{3}{*}{ P. aeruginosa 48B } & Allicin & 10 & 0 & 0 & 0 & 0 & 0 & 0 & 0 \\
\hline & Dimethyl thiosulfinate & 15 & 15 & 15 & 15 & 10 & - & - & - \\
\hline & Dipropyl thiosulfinate & 15 & 15 & 0 & 0 & 0 & 0 & 0 & 0 \\
\hline
\end{tabular}

*Thiosulfinate concentrations of $0.2,0.1$, and $0.05 \mathrm{mg} / \mathrm{ml}$ are not presented in the table, since no antibacterial effect was noted at these concentrations.

Table 3. Resistance $(+)$ of bacterial strains to antibiotics

\begin{tabular}{|c|c|c|c|c|c|c|c|c|c|c|c|c|c|c|c|c|c|}
\hline Strain & 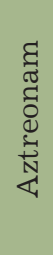 & 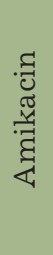 & 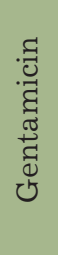 & 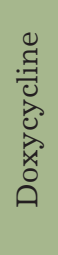 & 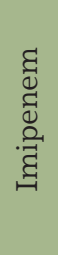 & 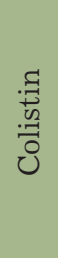 & 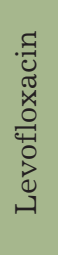 & 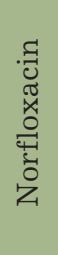 & 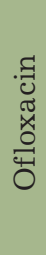 & 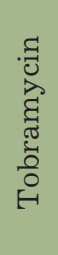 & 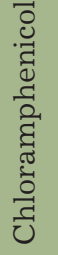 & 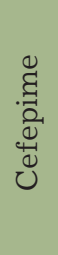 & 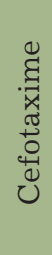 & 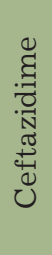 & 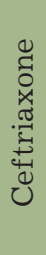 & 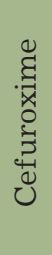 & 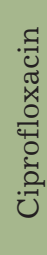 \\
\hline A. ruhlandii 155B & + & & + & + & + & + & + & + & + & + & + & + & + & + & + & + & + \\
\hline B. cenocepacia 122 & & + & + & & & & & & & + & & + & & + & & & + \\
\hline P. aeruginosa 48B & & & + & & & & + & & + & + & + & & + & + & + & & + \\
\hline
\end{tabular}

The MIC and MBC values for the effect of the thiosulfinates on $B$. cenocepacia were either equal or similar, thus indicating that these two compounds exhibit a bactericidal effect. The MIC value for allicin lies in the range obtained for the activity of commercial allicin against several strains of the $B$. cepacia complex $(0.008-0.062 \mathrm{mg} / \mathrm{ml})[11]$.

Thiosulfinates exhibit a bacteriostatic effect on $P$. aeruginosa 48B, since the $\mathrm{MBC}$ value in the studied range of thiosulfinate concentrations was determined only for allicin $(1 \mathrm{mg} / \mathrm{ml})$. The $\mathrm{MIC}$ and MBC values for the activity of allicin against $P$. aeruginosa $48 \mathrm{~B}$ correspond to the MIC $(0.064-0.512 \mathrm{mg} / \mathrm{ml})$ and MBC $(0.128-1.024 \mathrm{mg} / \mathrm{ml})$ values for the activity of allicin against the three clinical strains of $P$. aeruginosa [12].

The antibacterial effect of thiosulfinates on $A$. ruhlandii $155 \mathrm{~B}$ was the least significant. The MIC values obtained in the experiment for determining antibacterial activity on a solid growth medium using the disc diffusion method (Table 2) were $2 \mathrm{mg} / \mathrm{ml}$ for dimethyl and dipropyl thiosulfinates, which exceeded the maxi- mum concentration used in the serial dilution experiments. Allicin was the most effective thiosulfinate against $A$. ruhlandii 155B: it showed bactericidal action at a concentration of $1 \mathrm{mg} / \mathrm{ml}$.

Changes in the antibacterial efficacy of thiosulfinates were determined depending on the concentration of bacterial cells. The experiment was carried out using the disc diffusion method (Table 2) and by applying samples on a solid nutrient medium. The results obtained through both methods coincided.

Thiosulfinates at a concentration of $2 \mathrm{mg} / \mathrm{ml}$ effectively inhibited the growth of $A$. ruhlandii $155 \mathrm{~B}$ and B. cenocepacia 122 at a cell concentration $\leq 10^{7} \mathrm{CFU} / \mathrm{ml}$. The antibacterial effect of thiosulfinates against $P$. aeruginosa 48B was quite low. Allicin at maximum concentration only slightly suppressed the growth of $P$. aeruginosa $48 \mathrm{~B}$ even at minimal cell concentration. Interestingly, it was only dimethyl thiosulfinate that, among all thiosulfinates, suppressed the growth of $P$. aeruginosa at a concentration of $0.4 \mathrm{mg} / \mathrm{ml}$ (Table 2). The results obtained for allicin and dimethyl thiosul- 
Table 4. Antibacterial efficacy of thiosulfinates and antibiotics at a cell concentration of $10^{6} \mathrm{CFU} / \mathrm{ml}$

\begin{tabular}{|c|c|c|c|c|}
\hline \multirow{2}{*}{$\begin{array}{c}\text { Concentration, } \\
\mu \mathrm{g} / \text { disc }\end{array}$} & Thiosulfinate & \multicolumn{3}{|c|}{ Inhibition zone diameter, mm } \\
\cline { 2 - 5 } & & A.ruhlandii 155B & B. cenocepacia 122 & P. aeruginosa 48B \\
\hline 20 & Allicin & 25 & 20 & 0 \\
\hline 20 & Dimethyl thiosulfinate & 16 & 30 & 30 \\
\hline 20 & Dipropyl thiosulfinate & 30 & 5 & 0 \\
\hline 5 & Imipenem & 0 & 30 & 0 \\
\hline 10 & Tobramycin & 0 & 0 & 0 \\
\hline 10 & Ciprofloxacin & 0 & 0 & \\
\hline
\end{tabular}

finate are consistent with the data for $P$. aeruginosa from murine intestinal [7].

The absence of inhibition zones in the experiment on the solid growth medium with allicin and dimethyl thiosulfinate at low concentrations is probably due to the slow diffusion of substances into Muller-Hinton agar. Thus, the serial dilution method is the optimal technique for determining the antibacterial activity of the studied thiosulfinates.

The resistance of the A. ruhlandii 155B, B. cenocepacia 122 , and $P$. aeruginosa $48 \mathrm{~B}$ strains was evaluated using the 17 antibiotics most commonly prescribed to patients with cystic fibrosis (Table 3). The A. ruhlandii 155B strain showed resistance to 16 antibiotics, while the B. cenocepacia 122 and P. aeruginosa 48B strains were resistant to six and nine antibiotics, respectively. The obtained data confirmed that these strains develop resistance after prolonged antibiotic therapy. It is noteworthy that none of the antibiotics tested in this study had an antibacterial effect against all three bacterial strains.

We compared the efficacy of the antibacterial action of the thiosulfinates and broad-spectrum antibiotics belonging to the three different groups most commonly prescribed to patients with cystic fibrosis: imipenem belonging to the group of carbapenems, tobramycin belonging to the group of aminoglycosides, and cip- rofloxacin belonging to the group of fluoroquinolones (Table 4). Identically to the case of two-fold serial dilutions, determination of antibacterial activity by the method of disc diffusion on a dense growth medium demonstrated that three strains were resistant to tobramycin and ciprofloxacin at standard concentrations of $10 \mu \mathrm{g} /$ disc. The diameters of inhibition zones for B. cenocepacia 122 and $P$. aeruginosa $48 \mathrm{~B}$ were similar to those for dimethyl thiosulfinate and slightly higher than the inhibition zone of allicin for B. cenocepacia 122 . Allicin and dimethyl thiosulfinate inhibit the growth of A. ruhlandii 155B, while this strain is resistant to imipenem.

The obtained data open up possibilities for the development of agents for the antibacterial therapy of chronic pulmonary infections in patients with cystic fibrosis.

The authors would like to thank the staff of the N.F.Gamaleya National Research Centre of Epidemiology and Microbiology for the opportunity to conduct experiments on determining the antibacterial activity of drugs on clinical isolates.

The study was supported by the Russian Science Foundation (grant No. 15-14-00009).
REFERENCES

1. Shaginyan I.A., Kapranov N.I., Chernukha M.Yu., Alekseeva G.V., Semykin S.Yu., Avetisyan L.R., Kashirskaya N.Yu., Pivkina N.V., Danilina G.A., Batov A.B., Busuek G.P. // Journal of microbiology, epidemiology and immunobiology (Russian). 2009. № 5. P. 15-20.

2. Cavallito C.J., Bailey J.H. // J. Am. Chem. Soc. 1944. V. 66. P. 1950-1951.

3. Rabinkov A., Miron T., Konstantinovski L., Wilchek M., Mirelman D., Weiner L. // Biochim. Biophys. Acta. 1998. V. 1379. P. 233-244.

4. Muller A., Eller J., Albrecht F., Prochnow P., Kuhlmann K., Bandow J.E., Slusarenko A.J., Leichert L.I.O. // J. Biol. Chem. 2016. V. 291. P. 11477-11490.

5. Ankri S., Mirelman D. // Microb. Infect. 1999. V. 2. P. 125-129.

6. Morozova E.A., Kulikova V.V., Rodionov A.N., Revtovich
S.V., Anufrieva N.V., Demidkina T.V. // Biochimie. 2016. V. 128-129. P. 92-98.

7. Kulikova V.V., Anufrieva N.V., Revtovich S.V., Chernov A.S., Telegin G.B., Morozova E.A., Demidkina T.V. // IUBMB Life. 2016. V. 68. P. 830-835.

8. Miron T., Rabinkov A., Mirelman D., Weiner L., Wilchek M. // Anal. Biochem. 1998. V. 265. P. 317-325.

9. Mironov A.N., Bunatyan N.D. et al. Guidelines for preclinical trials of drugs. Part one. M.: Grif and K, 2012. 944 p.

10. Methodological guidelines 4.2.1890-04 Determination of the sensitivity of microorganisms to antimicrobial drugs (version 2015-02).

11. Wallock-Richards D., Doherty C.J., Doherty L., Clarke D.J., Place M., Govan J.R.W., Campopiano D.J. // PLoS One. 2014. V. 9. № 12 . e112726.

12. Reiter J., Levina N., van der Linden M., Gruhlke M., Martin C., Slusarenko A.J. // Molecules. 2017. V. 22. P. 1711. 